\title{
Current influences on adolescents' food choices within the school setting and opportunities for improvement: a qualitative study
}

\author{
L.D. Devine ${ }^{1}$, A.M. Gallagher ${ }^{1}$ and A.J. Hill ${ }^{1}$ \\ ${ }^{1}$ Nutrition Innovation Centre for Food and Health, School of Biomedical Sciences, Ulster University, Coleraine, \\ Northern Ireland
}

Current evidence indicates that UK adolescents' dietary intakes are suboptimal, with food consumed during the school day a particularly prevalent concern ${ }^{(1)}$. Promoting positive dietary behaviours within this age group is imperative to reduce the risk of future illhealth, as poor eating habits established during adolescence tend to track into adulthood ${ }^{(2)}$. The aim of this research was to investigate adolescents' perspectives on the key factors that influence their dietary choices within the school canteen and explore strategies on how best to encourage this population to select healthier options within this setting.

86 pupils in Year 8 (11-12 years) and Year 9 (12-13 years) were recruited from 6 purposively sampled post-primary schools across Northern Ireland (NI). 37 pupils in Year 8 (13 male; 24 female) and 49 pupils in Year 9 (21 male; 28 female) took part in the study. Pupils were invited to participate in focus group discussions which were undertaken in the school setting during May and June 2021. 13 mixed- gender focus groups (6 Year 8; 7 Year 9) were conducted with 5-8 participants per group. Informed assent and consent were obtained from pupils and their parents/ guardians respectively prior to participating in the study. Pupils' views and experiences of school meals, current influences on food choice within the school canteen at lunchtime and potential school-based opportunities to promote improved dietary choices among this age group were elicited using a semi-structured topic guide.

Focus group discussions ranged between 12 to 43 minutes (average 30 minutes) and were audio- recorded, transcribed verbatim and analysed thematically in six key phases ${ }^{(3)}$. NVivo12 software was used to facilitate the management of data. Ethical approval for this study was granted by Ulster University's Research Ethics Committee.

Six themes were identified for current factors influencing adolescents' food choices within the school canteen, namely: time and convenience (length of queues, time outside, grab-and-go options), peer influence (social acceptability), physical environment (location of food items, ease of access, food displays, limited menu, pricing and nutritional information), financial considerations (cost, portion size, satiety value), individual preferences (appearance, taste, familiarity) and food provision (food availability). Seven themes were also generated for strategies to improve food choice, namely: labelling (nutritional and ingredient labelling, point of choice labelling, school menu labelling), placement manipulations (healthy items displayed in prominent locations), special offers, pre-order facilities, menu and pricing displays, autonomy in food choice and incentives (social, financial and recognition rewards).

These results, together with findings from qualitative work with school stakeholders (principals, vice- principals, teachers, caterers, senior staff in the Education Authority) ${ }^{(4)}$ informed the design of a school-based dietary intervention tailored to the adolescent population which will be delivered and evaluated in post-primary schools across NI in 2021/22.

\section{Acknowledgments}

The authors thank the participating schools and pupils for their involvement in this study. This study was undertaken as part of a PhD scholarship funded by the Department for the Economy (DfE).

\section{References}

1. Taher AK, Ensaff H \& Evans CE (2020) Prev Med Rep 19, 101133.

2. Craigie AM, Lake AA, Kelly SA et al. (2011) Maturitas 70, 266-284.

3. Braun V \& Clarke V (2006) Qual Res Psychol 3, 77-101.

4. Devine LD, Gallagher AM \& Hill AJ (In Press) Proc Nutr Soc. 